\title{
WEBLABS: A VIRTUAL LABORATORY FOR COLLABORATIVE E-LEARNING
}

Evgenia Sendova, $\mathrm{PhD}^{1}$, Iliana Nikolova $\mathrm{PhD}^{2}$, George $\mathrm{Gachev}^{2}$ and Liliana Moneva $^{1}$

${ }^{1}$ Bulgarian Academy of Science. E-mail: jenny@math.bas.bg, lylymo@yahoo.com;

${ }^{2}$ University of Sofia, Bulgaria. E-mail: \{iliana, gachev\}@fmi.uni-sofia.bg

Abstract: The paper presents research carried out within WebLabs, a European project, where researchers, teachers and young students learn collaboratively in a technology enhanced environment. Experiences gained by the Bulgarian participants are discussed from an e-learning perspective.

Key words: visual programming; web-reports; collaborative e-learning; multiple representations.

\section{THE WEBLABS PROJECT}

Recent educational research emphasizes on "knowledge put into functional use in a multitude of different situations and contexts" [1]. Many educators are working in the filed of "new cultures of assignments", "project-oriented work" and are very interested in constructing environments in which the learners can actively work on a conceptual level [2]. This is especially true in the case of mathematics taught at junior high school level where the mathematical concepts are often "shadowed" by the necessities of calculating, symbol manipulating by hand, drawing, etc. Restricting the math education to these technical skills creates a very twisted image of mathematics and the young learners can hardly experience the real spirit of mathematics as a research field.

To cultivate abilities of young learners to generate, test, play with ideas and to build a culture of communicating them to their peers from different countries is one of the fundamental goals of the WebLabs project [3]. The 
project aims at creating a virtual laboratory and a community of young learners, teachers and researchers exploring collaboratively mathematical and science phenomena.

\subsection{The focus of WebLabs}

This is a 3 year EU-funded educational research project carried out by scientific and educational institutions of Bulgaria, Cyprus, Italy, Portugal, Sweden and UK. It creates a new representational infrastructure for elearning. The focus is on collaborative construction, description and interpretation of important mathematical and science concepts by means of modern ICTs. A web-based environment, wplone, is used to mediate collaboration so that the participants can share ideas and constructively criticize each other's evolving knowledge and working models [4]. The project embraces exploratory activities in the following domains: mathematics, kinematics and dynamics, complex biological and ecological systems, robotics.

\subsection{The computer environment Toon Talk}

WebLabs uses an environment for visual programming called Toon Talk in which the source code is animated thus allowing for abstract computational concepts to be represented by concrete analogues, instantiated in cartoon-like characters [5]. ToonTalk has some unique features suitable for visualizing and exploring mathematics concepts and ideas when working with junior-high school students. The mathematical activities are integrated in a natural way with cultivating some programming skills. The programs in ToonTalk take the form of animated robots, which can be named, picked up and trained to perform a certain sequence of elementary steps. After the training, the robots runforever if the initial conditions are satisfied. Such type of programming is especially appealing to young programmers since it is amusing, requires creativity and stimulates the acquiring of new skills for solving problems.

\subsection{Developing a collaborative community}

The students in WebLabs have the chance of working together not only with their peers from other countries, but also with distinguished researchers. Rather than being just reality check for researchers they are real partners in a research process, in which new learning methods and computer technologies are used and experimented with. When using ToonTalk as a 
means for modeling the students learn how to work with contemporary computer environments in a natural way. In the context of carefully designed educational activities they gain knowledge about important processes and phenomena from mathematics, physics, biology, and compare their understanding with the rest of the participants. The communication is carried out by the so-called Webreports - a specially designed concept enabling the young learners to share and discuss the problems they have solved, and even more interesting - the problems they have formulated and implemented by means of ToonTalk robots.

\subsection{WebLabs e-learning elements}

The WebLabs project embraces various elements of e-learning. The specific means through which they are implemented are:

- long distance birds: a ToonTalk concept for information exchange among different computers;

- Web-reports: a mechanism for the participants to describe and share their ideas and programming constructs; a visual on-line editor is available to compose web-reports on individual or group work, to add comments and publish working ToonTalk models of students' ideas as they develop;

- Plone: a web-based environment used to mediate the collaborative learning activities over distance.

What follows, are excerpts from web-reports written by WebLabs students from Sofia and reflecting two class sessions on number sequences.

\subsection{Weblabetics - a children's endeavor for a better communication in an international context}

Excerpts from a group web-report [6]:

When browsing through plone in search for interesting sequences we had very unexpected experience. We moved step-by-step through the sequences suggested by Nikmous, Kiriakos, Irakli - all in Greek. The sequences are very clear but when the comments are in a language, which we don't understand, it is very annoying. So George, our teacher, asked us: Can you think of a way to express ToonTalk ideas so that anyone could understand them? Yana suggested to use pictures for representing the ToonTalk characters and drew some on the board (fig. 1): 


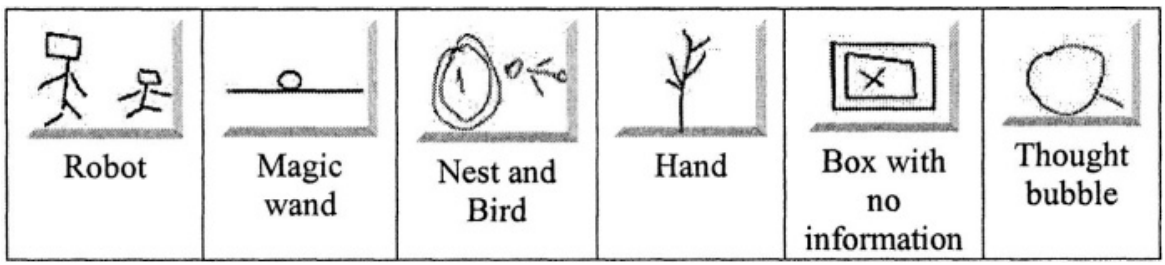

Figure 1. Pictures representing ToonTalk tools

George challenged us to translate our Counting Robot in the new language. We all thought that this was easy but soon realized that we didn 't have symbols for actions in our alphabet (or rather - weblabetics). So we added arrows for "puts" and "takes". Here is the Counting Robot in weblabetics (fig. 2):

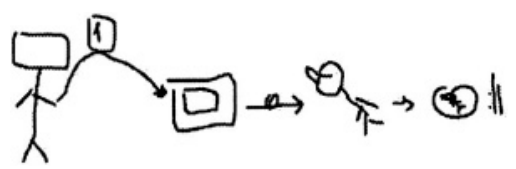

Figure 2. Program code (Counting Robot) in weblabetics

Isn't this clear for everybody? Well, just in case you lack the experience:

A robot puts 1 in a box, then copies the content, gives it to a bird, which puts it in its nest. Afterwards everything is repeated. Do you see the ":\|" sign at the end -this is the music symbol for a repetition-Peter thought of it! In short, this is our old friend - the Counting Robot (in new clothes...)

We hope that now it would be easier to talk about ToonTalk and our ideas to everyone in the WebLabs project.

George told us the story of the Babylonian tower - a common language for everyone is more effective than many languages for a few.

Reflection: Children were faced with a typical e-learning problem while trying to learn collaboratively over distance - the language problem. In an attempt to overcome it, they reached the idea of designing a graphical scripting language for visual programming.

\subsection{When the math gauntlet is thrown from a distance}

After hearing from Yishay Mor, a researcher from the UK WebLabs team, that there is a new challenging sequence on the "Guess my robot" page [7] published by a Portuguese girl (Rita), the Sofia teachers (George and Jenny) asked the students to solve it as a homework. Two students, Nasko 
and Ivan, took the challenge and reported to the class that they had guessed the rule and had even built robots generating it. The teachers decided to use this as a basis for the topic "Different representations of number sequences" [8].

Jenny: Nasko, please try to translate your robot in algebraic language?

Nasko: Here is the relationship between the consecutive terms (fig. 3)

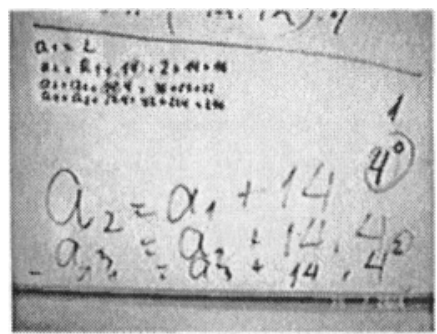

Figure 3. In algebraic language

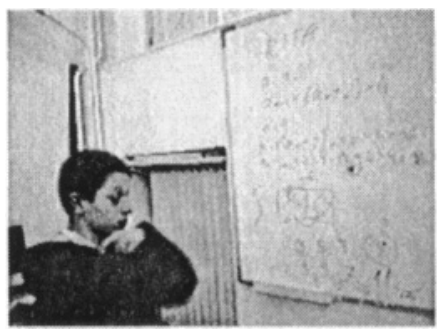

Figure 4. In "Robot" language

Vessela: Ah, it means we have to present 1 as $4^{0}$

Jenny: Rita's robot in algebraic language looks like this:

$a_{1}=2, a_{n}=4\left(a_{n-1}+2\right)$. Are these sequences the same?

Teddy: Ah, this looks much easier. Let me try to translate it in a "robot language" (Fig. 4)

Jenny: Do you think that your and Nasko's robot will produce the same sequence?

Teddy: Sure! Absolutely!

Jenny: How do you know? Take for example the sequence 3, 5, 7, ... Which is the next term?

Teddy: 9, of course!

Jenny: Do you agree, George?

George: I am thinking of 11.

Jenny: Of course! (Both of us are thinking of a subsequence of the prime numbers (of course!!!))

Teddy: Oh, I see, so you could extend the sequence in more than one way... - adding twice 2, and then adding twice 4, etc. So, I could write it in algebraic way as follows: $a_{n}=a_{n \cdot 1}+2^{n}$

Vessela: What about $a_{n}=a_{n-1}+a_{n-2}-1$ ?

Ivan: $M y$ robot also uses 2 previous terms : $a_{n+1}=a_{n}+\left(a_{n}-a_{n-1}\right) * 4$ and its first numbers coincide with Rita's ones.

Teddy: Oh, how could we compare so many robots? 
Jenny: I am not telling you! But I hope to hear your ideas next time. Please consider for the following questions from our English partners [9]:

1. How did you guess the rule of Rita's sequence?

2. Which is easier for you - to translate from the robot language into algebraic one, or vice versa?

3. How could we check if two robots produce the same sequence?

Reflections: In a panel discussion on the educational value of computer programming diSessa [10] proposes the idea that the intellectual power the programming representations can have for learning science is at least comparable to, if not greater than, algebra. We can easily adopt it in the context of learning mathematics - gaining the flexibility of moving from a programming to algebraic representation of a sequence contributes to a deeper understanding of the mathematical ideas.

An important element for a good learning is the students' motivation - in our case the problem was formulated as a challenge by a peer of theirs. As for the teacher, s/he is seen as a facilitator, as a guide in a discovery process, providing new tools only when needed.

\subsection{Conclusions}

The effect of working with young students in the framework of WebLabs is not reduced to learning specific aspects of certain subject areas but has a much larger scope. On one hand, the scientist in the learner is enhancedthe students get used to pose questions, to look for answer no matter how sophisticated they might be. They develop an understanding of mathematics as a science in which formulating hypotheses, carrying out experiments, and attacking open problems plays a crucial part. The students are partners in a research process and can influence both the development of the computer environment and the design of the educational activities. They can communicate among themselves, with teachers and researchers locally and globally alike. During this communication they acquire specific social experience and are stimulated to build valuable personal skills and abilities, such as:

- to generate and verbalize ideas;

- to present their results according to a concrete standard;

- to share their experience by means of electronic communication;

- to discuss their work and to work in a team;

- to be (self-)critical to the work published in the virtual environment 


\section{ACKNOWLEDGEMENT}

We acknowledge the support of the WebLabs project: "WebLabs: new representational infrastructures for e-learning"; contract number IST 20013220

\section{REFERENCES}

1. ICMI, Study 14, Application and Modeling in Mathematics Education, Discussion Document, 2003

2. Loethe, H. Concept Oriented Learning Environments: a Project Using Sequences in Scheme, in "Be Creative...re-inventing technology on education", Proceedings of Eurologo 2003, Portugal

3. WebLabs http://www.weblabs.eu.com

4. Mor, Y, Sendova, E. ToonTalking about mathematics, in Proceedings of the International Congress MASSEE 2003, p.36-43

5. Toon Talk http:// www.toontalk.com

6. http://www.weblabs.org.uk/wlplone/Members/Sofia/my_reports/Report.2004-04-13.0957

7. http://www.weblabs.org.uk/wlplone/Members/Rita/

8. http://www.weblabs.org.uk/wlplone/Members/Sofia/my_reports/Report.2004-02-26.2431

9. Mor et al, Thinking in Process, (to appear in MicroMath)

10.Klopfer, E. et al. Programming Revised - The Educational Value of Computer Programming, http://www.cs.berkeley.edu/ abegel/papers/icls04.pdf 